Agriculture

Agriculture fields

Okayama University

Year 2002

\title{
Morphological features of lipid droplet transition during porcine oocyte fertilisation and early embryonic development to blastocyst in vivo and in vitro
}

\author{
Kazuhiro Kikuchi* ${ }^{*}$ Hans Ekwall ${ }^{\dagger} \quad$ Paisan Tienthai $^{\ddagger}$ \\ Yasuhiro Kawai** Junko Noguchi ${ }^{\dagger \dagger}$ \\ Hiroyuki Kaneko ${ }^{\ddagger \ddagger} \quad$ Heriberto Rpdriguez-Martinez ${ }^{\S}$
}

* Swedish University of Agricultural Sciences

${ }^{\dagger}$ Swedish University of Agricultural Sciences

${ }^{\ddagger}$ Swedish University of Agricultural Sciences

** Okayama University

${ }^{\dagger}$ National Institute of Agrobiological Sciences

$\ddagger \ddagger$ National Institute of Agrobiological Sciences

$\S$ Swedish University of Agricultural Sciences

This paper is posted at eScholarship@OUDIR : Okayama University Digital Information Repository.

http://escholarship.lib.okayama-u.ac.jp/agriculture_general/4 


\title{
Morphological features of lipid droplet transition during porcine oocyte fertilisation and early embryonic development to blastocyst in vivo and in vitro
}

\author{
Kazuhiro Kikuchi $i^{1,2}$, Hans Ekwall ${ }^{3}$, Paisan Tienthai ${ }^{1}$, Yasuhiro Kawai ${ }^{1,4}$, Junko Noguchi ${ }^{2}$, Hiroyuki \\ Kaneko ${ }^{2}$ and Heriberto Rodriguez-Martinez ${ }^{1}$ \\ Swedish University of Agricultural Sciences, Uppsala, Sweden, National Institute of Agrobiological Sciences, Tsukuba, Japan \\ and Okayama University, Okayama, Japan
}

Date submitted: 5.5.02. Date accepted: 25.7.02

\section{Summary}

Lipid content in mammalian oocytes or embryos differs among species, with bovine and porcine oocytes and embryos showing large cytoplasmic droplets. These droplets are considered to play important roles in energy metabolism during oocyte maturation, fertilisation and early embryonic development, and also in the freezing ability of oocytes or embryos; however, their detailed distribution or function is not well understood. In the present study, changes in the distribution and morphology of porcine lipid droplets during in vivo and in vitro fertilisation, in contrast to parthenogenetic oocyte activation, as well as during their development to blastocyst stage, were evaluated by transmission electron microscopy (TEM). The analysis of semi-thin and ultra-thin sections by TEM showed conspicuous, large, electron-dense lipid droplets, sometimes associated with mitochondrial aggregates in the oocytes, irrespective of whether the oocytes had been matured in vivo or in vitro. Immediately after sperm penetration, the electron density of the lipid droplets was lost in both the in vivo and in vitro oocytes, the reduction being most evident in the oocytes developed in vitro. Density was restored in the pronculear oocytes, fully in the in vivo specimens but only partially in the in vitro ones. The number and size of the droplets seemed, however, to have decreased. At 2- to 4-cell and blastocyst stages, the features of the lipid droplets were almost the same as those of pronuclear oocytes, showing a homogeneous or saturated density in the in vivo embryos but a marbled or partially saturated appearance in the in vitro embryos. In vitro matured oocytes undergoing parthenogenesis had lipid droplets that resembled those of fertilised oocytes until the pronuclear stage. Overall, results indicate variations in both the morphology and amount of cytoplasmic lipid droplets during porcine oocyte maturation, fertilisation and early embryo development as well as differences between in vivo and in vitro development, suggesting both different energy status during preimplantation development in pigs and substantial differences between in vitro and in vivo development.

Keywords: Culture, In vitro, In vivo, Lipid droplet, Pig

All correspondence to: K. Kikuchi, DVM, PhD, Genetic Diversity Department, National Institute of Agrobiological Sciences, Kannondai 2-1-2, Tsukuba, Ibaraki 305-8602, Japan. Tel: +81 29838 7447. Fax: +81 29838 7408. e-mail: kiku@nias.affrc.go.jp

${ }^{1}$ Departments of Obstetrics and Gynaecology, Faculty of Veterinary Medicine, Swedish University of Agricultural Sciences (SLU), SE-750 07 Uppsala, Sweden.

${ }^{2}$ Genetic Diversity Department, National Institute of Agrobiological Sciences (NIAS), Tsukuba, Ibaraki 305-8602, Japan. ${ }^{3}$ Department of Anatomy and Histology, Faculty of Veterinary Medicine, Swedish University of Agricultural Sciences (SLU), SE-750 07 Uppsala, Sweden.

${ }^{4}$ Department of Animal Science, Faculty of Agriculture, Okayama University, Okayama 700-8530, Japan.

\section{Introduction}

Lipid droplet content in mammalian oocytes or embryos differs among species. Bovine and porcine oocytes and embryos usually show large amounts of lipid droplets, in contrast to rodent or human oocytes. Lipid droplets are considered to be a source of energy (Brown, 2001) and seem to play important roles in oocyte maturation, fertilisation and development to embryos. Lipid content rates, furthermore, to higher sensitivity to freezing during cryopreservation of embryos in cows (Mohr \& Trounson, 1981; Leibo \& Loskutoff, 1993; Yamashita et al., 1999; Abe et al., 2002) 
and pigs (Nagashima et al., 1995). Although freezing of porcine embryos derived in vivo has been successful (reviewed by Dobrinsky, 2002), there are no reports describing successful cryopreservation of in vitro produced embryos. Although the viability of in vitro embryos is considered to be lower than that of in vivo ones, it is possible that a difference in their lipid contents results in a different sensitivity to freezing. However, little is known about lipid droplet distribution or function throughout maturational and developmental stages, or about the difference between in vivo and in vitro materials.

It is possible to detect cytoplasmic lipids. Methods have been established to measure the content of lipid or fatty acids in bovine oocytes or embryos (Ferguson \& Leese, 1999; Sata et al., 1999; McEvoy et al., 2000; Kim et al., 2001). For example, the lipid content and fatty acid composition of the total lipid fraction has been analysed by gas chromatography in matured oocytes (Kim et al., 2001). On the other hand, morphological evaluation of lipid droplet distribution seems to be a rather simple method to determine their content and follow changes in localisation during development. Lipid deposits often accumulate in large masses, being defined as 'lipid droplets', which can be easily observed in sections. Use of transmission electron microscopy (TEM) procedures reveals droplets of high electron density following fixation with osmium tetroxide. Ultrastructural analyses of bovine (Plante \& King, 1994; Abe et al., 1999a, b; Crosier et al., 2001) and porcine (Hyttel \& Niemann, 1990) embryos up to the blastocyst stage have been performed. However, detailed information about transient changes in lipid droplets during embryo development is rather scarce (bovine: Abe et al., $1999 a, b)$. The present study was undertaken to determine the morphological changes in lipid droplets during porcine fertilisation, parthenogenetic activation and early embryonic development in vivo and in vitro, using sections evaluated by light microscopy and TEM.

\section{Materials and methods}

\section{In vivo-developed gametes, zygotes and embryos}

All in vivo-developed gametes, zygotes and embryos were collected from normally cycling multiparous cross-bred (Swedish Yorkshire $\times$ Swedish Landrace) sows, recruited for the experiments after weaning, and individually penned at the Department of Obstetrics and Gynaecology, SLU, Uppsala. The sows received standard ration and water ad libitum, according to Swedish standards (Simonsson, 1994). Fertile boars were always penned in the vicinity. The sows were checked twice daily by experienced personnel for behavioural oestrus. Six sows (out of 8) were randomly mated twice (at $12 \mathrm{~h}$ and 24 after onset of oestrus) with one of two fertile boars. Occurrence of ovulation was recorded by transrectal ultrasonography (TUS) using an annular array scanner (Scanner 250, Pie Medical, Maastricht, The Netherlands) with a $5 \mathrm{MHz}$ multipleangle transducer. A specially constructed wagon was used to immobilise the sows during TUS scanning (Mburu et al., 1995). The experimental design was reviewed and approved by the Ethics Committee for Experimentation with Animals in Sweden.

Follicular oocytes were collected from two sows that showed onset of oestrus about $36 \mathrm{~h}$ prior to slaughter. After denudation of cumulus cells by hyaluronidase treatment and gentle pipetting, oocytes with the first polar body were categorised as in vivo-matured oocytes. Ovulated oocytes were collected at approximately $5 \mathrm{~h}$ after ovulation from a mated sow. These oocytes were categorised as in vivo-fertilised oocytes. At $10 \mathrm{~h}$ after the confirmation of ovulation, oviductal oocytes were also collected from another two mated sows. They were categorised as in vivo pronuclear oocytes. Some of the oocytes from these groups were fixed and evaluated for nuclear status. On the second day after ovulation, cleaved embryos were collected as in vivo early embryos from a mated sow, and on the sixth day, blastocysts were also collected as in vivo blastocysts from the other two sows. Five to 12 oocytes or embryos were collected from each sow.

\section{In vitro maturation (IVM), fertilisation (IVF) and culture (IVC)}

All the in vitro materials were obtained as described previously (Kikuchi et al., 1999a, 2002). In brief, porcine ovaries from pre-pubertal Large White gilts were obtained at a local slaughterhouse and transported to the laboratory at $35{ }^{\circ} \mathrm{C}$. Cumulus-oocyte complexes (COCs) were collected from follicles $3-5 \mathrm{~mm}$ in diameter in a collection medium that consisted of Medium 199 (with Hanks' salts; Gibco, Life Technologies, Grand Island, NY) supplemented with 10\% (v/v) fetal bovine serum (Gibco), $20 \mathrm{mM}$ HEPES (Dojindo Laboratories, Kumamoto, Japan), $100 \mathrm{IU} / \mathrm{ml}$ penicillin $\mathrm{G}$ potassium (Sigma Chemical, St Louis, MO) and 0.1 $\mathrm{mg} / \mathrm{ml}$ streptomycin sulfate (Sigma) (Kikuchi et al., 1993). About 30 COCs were cultured in each $500 \mu \mathrm{l}$ of maturation medium, a modified NCSU-37 solution (Petters \& Wells, 1993; Funahashi et al., 1997) containing $10 \%(\mathrm{v} / \mathrm{v})$ porcine follicular fluid, $50 \mu \mathrm{M} \beta$-mercaptoethanol, $0.6 \mathrm{M}$ cysteine, $1 \mathrm{M}$ dibutytyl cAMP (dbcAMP, Sigma), 10 IU / ml eCG (PMS 1000 IU, Nihon Zenyaku Kogyo, Koriyama, Japan) and 10 IU/ml hCG (Puberogen 500 unit, Sankyo, Tokyo, Japan), in 4-well dishes (Nunclon Multidishes, Nalge Nunc International, Denmark) for 20-22 h. They were subsequently cultured in the maturation medium without 
dbcAMP and hormones for $24 \mathrm{~h}$. The maturation culture was carried out under conditions of $\mathrm{O}_{2} \mathrm{CO}_{2}$ and $\mathrm{N}_{2}$ adjusted to $5 \%, 5 \%$ and $90 \%$, respectively, at $39^{\circ} \mathrm{C}$.

After denudation, oocytes with the first polar body were collected as in vitro-matured oocytes. Frozen and thawed epididymal spermatozoa (Kikuchi et al., 1998) from a Landrace boar were preincubated for $1 \mathrm{~h}$ at $37^{\circ} \mathrm{C}$ in Medium 199 adjusted to pH 7.8 (Nagai et al., 1988). Fertilisation medium for porcine oocytes (Pig-FM) (Suzuki et al., 2000) consisting of $90 \mathrm{mM} \mathrm{NaCl}, 12 \mathrm{mM}$ $\mathrm{KCl}, 25 \mathrm{mM} \mathrm{NaHCO}, 0.5 \mathrm{mM} \mathrm{NaH} \mathrm{PO}_{4}, 0.5 \mathrm{mM}$ $\mathrm{MgSO}_{4}, 10 \mathrm{mM}$ sodium lactate and $10 \mathrm{mM}$ HEPES was modified by Suzuki (Suzuki et al., 2002) by the addition of $8 \mathrm{mM} \mathrm{CaCl} 2,2 \mathrm{mM}$ sodium pyruvate, $2 \mathrm{mM}$ caffeine and $5 \mathrm{mg} / \mathrm{ml}$ bovine serum albumin (BSA; Fraction $\mathrm{V}$, Sigma). A portion $(10 \mu l)$ of the preincubated spermatozoa was introduced into $90 \mu \mathrm{l}$ fertilisation medium containing about 20 COCs surrounded by expanded cumulus cells. The final sperm concentration was adjusted to $1 \times 10^{5}$ cells $/ \mathrm{ml}$. Co-incubation was carried out for $3 \mathrm{~h}$ at $39^{\circ} \mathrm{C}$ under $5 \% \mathrm{O}_{2}$. Then the oocytes were freed from the cumulus cells and attached spermatozoa.

Some oocytes were collected as in vitro-fertilised oocytes. The others were transferred into IVC-PyrLac medium (NCSU-37 medium without glucose, but containing $4 \mathrm{mg} / \mathrm{ml} \mathrm{BSA}, 50 \mu \mathrm{M} \beta$-mercaptoethanol, $0.17 \mathrm{mM}$ sodium pyruvate and $2.73 \mathrm{mM}$ sodium lactate), conditioned by the oviductal epithelial cells (Kikuchi et al., 2002), and subsequently cultured in vitro. Some inseminated oocytes at $10 \mathrm{~h}$ post-insemination and the 3- to 4-cell stage embryos on the second day (day 2) were collected as in vitro pronuclear oocytes and in vitro early embryos, respectively. On day 2, the remaining embryos were transferred to IVC-Glu medium (NCSU-37 plus BSA and $\beta$-mercaptoethanol) and cultured for a further 4 days (until day 6). At day 6 , expanded blastocyst were harvested as in vitro blastocysts. The collections were carried out twice. A total of 70 oocytes or embryos for each category were harvested for evaluation by light microscopy and TEM.

\section{Parthenogenetic activation of IVM oocytes}

In vitro-matured oocytes were selected and stimulated as previously described (Kikuchi et al., 1995). They were transferred to activation solution consisting of $0.28 \mathrm{mM}$ $d$-mannitol (Wako Pure Chemical, Tokyo, Japan), 0.05 $\mathrm{mM} \mathrm{CaCl} 2 \mathrm{H}_{2} \mathrm{O}$ (Wako), $0.1 \mathrm{mM} \mathrm{MgCl}_{2} 2 \mathrm{H}_{2} \mathrm{O}$ (Wako) and $0.01 \mathrm{mg} / \mathrm{ml} \mathrm{BSA}$. They were transferred to the hybridising chamber (FTC-22W, Shimadzu, Tokyo, Japan) containing $50 \mu \mathrm{l}$ activation solution and stimulated with a $20 \mu \mathrm{s}$ pulse at $1.5 \mathrm{kV} / \mathrm{cm}$ DC using a somatic hybridiser (SSH-10, Shimadzu). The stimulated oocytes were subsequently cultured for $3 \mathrm{~h}, 10 \mathrm{~h}$ and 20 $\mathrm{h}$ and harvested as parthenogenetic stimulated oocytes $(3 \mathrm{~h})$ and parthenogenetic pronuclear oocytes (10 and
$20 \mathrm{~h})$, respectively. The collections were carried out twice. A total of 70 oocytes or embryos for each category were harvested for evaluation by light microscopy and TEM.

\section{Evaluation of oocyte/embryo stage under light microscopy}

For the confirmation of oocyte/embryo nuclear stage, some of the harvested oocytes or embryos (a total of 3-5 for in vivo materials and about 50 for in vitro materials in each category) were whole-mounted and fixed in acetic alcohol (1:3). After staining with $1 \%(\mathrm{w} / \mathrm{v})$ aceto-orcein solution, they were evaluated by a light microscope equipped with phase-contrast optics.

\section{Evaluation by TEM}

All the remaining oocytes and embryos were fixed in a $2.5 \%$ solution of glutaraldehyde in sodium cacodylate buffer (0.067 $\mathrm{M}, \mathrm{pH} 7.2-7.4)$ and stored in the same solution at $4{ }^{\circ} \mathrm{C}$ until being processed. After post-fixation for $5 \mathrm{~min}$ in $2 \%(\mathrm{w} / \mathrm{v})$ osmium tetroxide in the cacodylate buffer, specimens were embedded in Agar $^{100}$ resin (Agar Scientific, Cambridge, UK). Semithin section and continuous ultra-thin sections of oocytes and embryos were processed conventionally for TEM. Semi-thin sections for light microscopy were stained with toluidine blue. Thereafter, ultra-thin sections were cut and stained with uranyl acetate and lead citrate, and examined by a TEM (Philips 420 electron microscope, Einhoven, The Netherlands) at $80 \mathrm{kV}$. Five to seven oocytes or embryos for each category were evaluated.

\section{Results}

Developmental stages of all the categorised oocytes or embryos in the present study were confirmed with whole-mounted specimen (Table 1). The stages of the in vivo materials were almost uniform and synchronised in each collection. In vivo-fertilised oocytes were estimated to be at 3-6 h after gamete encounter since they were found to be at telophase II with the identified second polar body or at an early stage of pronucleus formulation. In vivo pronuclear oocytes were estimated to be at $4-20 \mathrm{~h}$ after gamete encounter due to the existence of well-developed male and female pronuclei. Two-cell stage embryos and hatched blastocysts were collected as in vivo early embryos and in vivo blastocyts, respectively. On the other hand, in vitro specimens were not at the same stage because all the oocytes were not matured and fertilised. In the present study, about $70 \%(37 / 53)$ of the cultured oocytes were matured and about $50 \%(18 / 37)$ of the matured oocytes 
Table 1 Developmental stages of oocytes or embryos as categorised in Materials and Methods

\begin{tabular}{|c|c|c|c|c|c|}
\hline \multirow[b]{2}{*}{$\begin{array}{l}\text { Oocyte/ } \\
\text { embryo }\end{array}$} & \multicolumn{5}{|c|}{ Categorised as: } \\
\hline & $\begin{array}{l}\text { Matured } \\
\text { oocyte }\end{array}$ & $\begin{array}{l}\text { Fertilised or } \\
\text { activated }^{a} \\
\text { oocyte }\end{array}$ & $\begin{array}{l}\text { Pronuclear } \\
\text { oocyte }\end{array}$ & $\begin{array}{l}\text { Early } \\
\text { embryo }\end{array}$ & Blastocyst \\
\hline In vivo & Metaphase II & $\begin{array}{l}\text { Telophase II or } \\
\text { early pronuclear } \\
\text { stage }\end{array}$ & $\begin{array}{l}\text { FPN and MPN } \\
\text { formed }\end{array}$ & $\begin{array}{l}\text { 2-cell } \\
\text { storage }\end{array}$ & $\begin{array}{c}\text { Hatched } \\
\text { blastocysts }\end{array}$ \\
\hline In vitro & Metaphase II & $\begin{array}{c}\text { Anaphase II or } \\
\text { telophase II }\end{array}$ & $\begin{array}{l}\text { FPN and MPN } \\
\text { formed }\end{array}$ & $\begin{array}{l}\text { 3- to } 4- \\
\text { cell } \\
\text { stage }\end{array}$ & $\begin{array}{l}\text { Expanded } \\
\text { blastocysts }\end{array}$ \\
\hline Parthenogenetic & $i c^{a}$ & Telophase II & $\begin{array}{c}\text { FPN formed } \\
\text { or fragmented oocytes }\end{array}$ & - & - \\
\hline
\end{tabular}

FPN, female pronucleus; MPN, male pronucleus.

an vitro-matured oocytes were stimulated with an electric pulse.

${ }^{b}$ Some fragmented oocytes were seen at $20 \mathrm{~h}$ after stimulation. In the present study, pronuclear oocytes at 10 and $20 \mathrm{~h}$ were collected.

were fertilised, where the monosperm fertilisation rate was about $30 \%$ (11/37). Most fertilised oocytes were at the anaphase II or telophage II stage at $3 \mathrm{~h}$ after insemination, and they formed both male and female pronuclei at $10 \mathrm{~h}$ (average number of penetrated spermatozoa was 3.2). We carefully checked for evidence of fertilisation - for example, extrusion of cortical granules, existence of the second polar body or pronucleus - during preparation of semi-thin sections for TEM. On day 2 of IVC, the percentage of 3- to 4-cell stage embryos was $42 \%$ (20/48). They were collected as in vitro early embryos. On day 6, the blastocyst formation rate was $20 \%(10 / 51)$. The expanded blastocysts were collected as in vitro blastocysts. As regards parthenogenesis, some of the stimulated oocytes were activated to telophase II stage at $3 \mathrm{~h}$ after the stimulation, and more than $80 \%$ (42/50) of the oocytes formed female pronuclei at $10 \mathrm{~h}$. Some fragmented oocytes existed but most of them were pronuclear oocytes even at $20 \mathrm{~h}$.

Semi-thin sections from matured oocytes, fertilised oocytes and embryos, and also parthenogenetic activated oocytes, are shown in Figs. 1-3, and ultra-thin sections in Figs. 4-6. The analysis of ultra-thin sections at TEM showed conspicuous, large, electron-dense lipid droplets, sometimes associated with mitochondrial aggregates in the oocytes, irrespective of these being matured in vivo or in vitro (Fig. $4 a$ and $d$, respectively). Lipid droplets in matured oocytes featured finely marbled or partially saturated figures, with an associated homogeneous area. The electron density of the lipid droplets was lost in both the in vivo and in vitro oocytes immediately after sperm penetration and the saturated structure was barely seen in either oocyte type (Fig. $4 b$ and $e$, respectively). The density of the lipid droplets in the in vivo-fertilised oocytes was reduced to pale grey, while the density seemed to be almost completely lost in the in vitro-fertilised oocytes. At the pronculear stage, electron density was restored fully in the in vivo specimens but only partially in the in vitro ones (Fig. $4 c$ and $f$, respectively). The structure in the in vivo droplets always appeared homogeneous in density, while that in the in vitro ones showed a distinct marbled or partially saturated appearance. These changes in lipid density were clearly observed in semithin sections as well (Fig. 1a-f).

During embryonic development, the electron density of the lipid droplets was high in both the in vivo and the in vitro embryos, whereas the number and size of the droplets in the in vitro specimens were larger than in those in vivo (Fig. $2 a-d$ ). The fine structure confirmed these observations (Fig. $5 a-d$ ). The droplets in the in vivo embryos were homogeneous with a higher electron density than the in vitro specimens. On the other hand, the droplets in the in vitro early embryos had similar features to those in the in vivo-matured or pronuclear oocytes. The in vitro-developed blastocysts had lipid droplets with a higher electron density than in the in vivo ones, albeit with a lower homogeneity. These features were observed in both inner cell mass and trophoblast cells.

When in vitro-matured oocytes were stimulated with electric pulses, the density of the droplets in both semi-thin and ultra-thin sections was not reduced by $3 \mathrm{~h}$ after the stimulation (Figs. $3 a$ and $6 a$, respectively). This feature was similar to that in the pronuclear oocytes both in vivo and in vitro, and this morphological appearance was almost the same until 10 or $20 \mathrm{~h}$ after stimulation in both semi-thin (Fig. $3 b$ and $c$, respectively) and ultra-thin (Fig. $6 b$ and $c$, respectively) sections. 


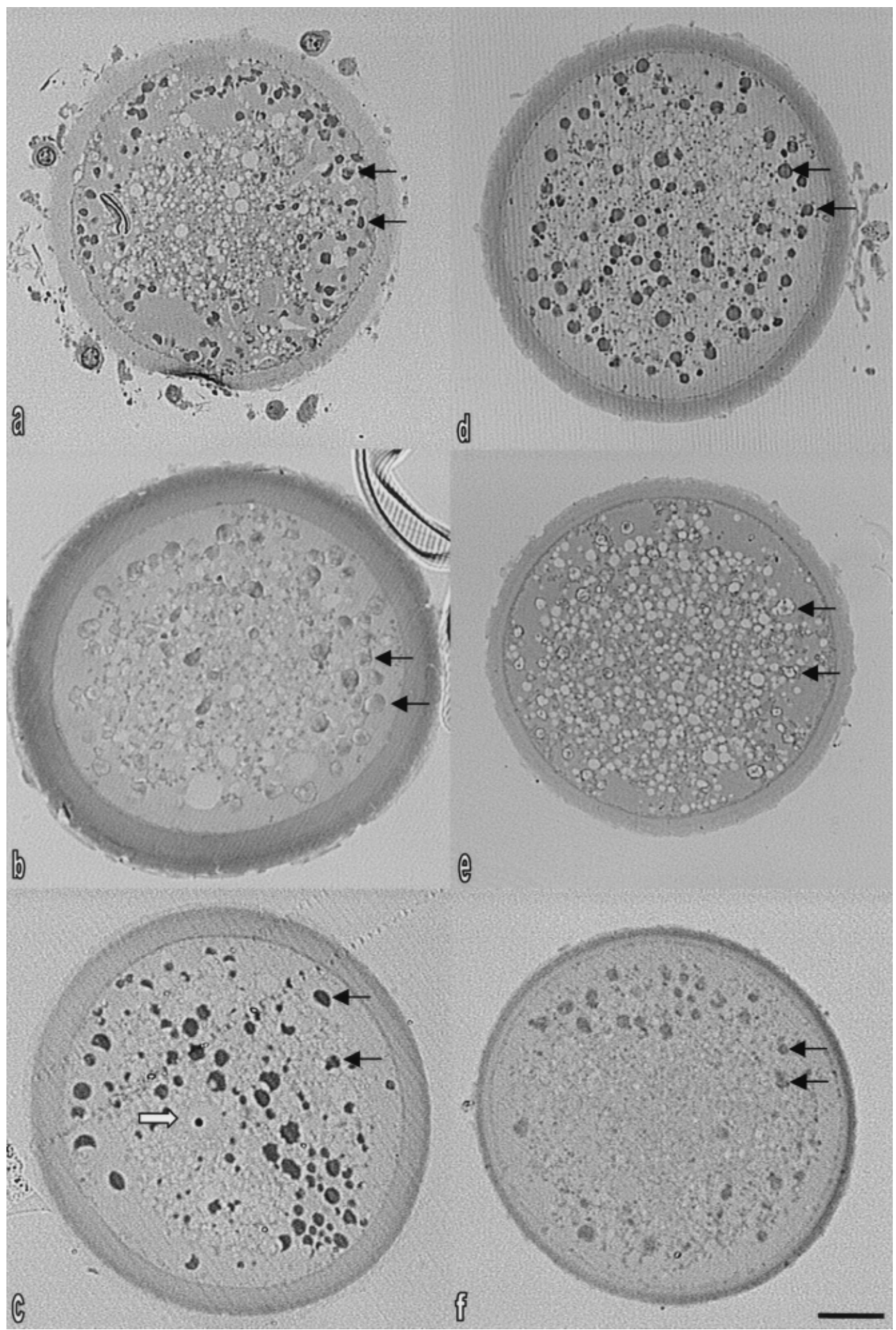

Figure 1 Light micrographs of semi-thin sections of porcine oocytes in vivo $(a-c)$ or in vitro $(d-f)$ matured $(a, d)$, fertilised $(b, e)$ or at the pronuclear stage $(c, f)$ depicting lipid droplet (small arrows) localised throughout. Note the lower density of the lipid droplets at fertilisation compared with matured oocytes, and the restoration of the higher density at the proncuclear stage. White arrow, pronucleus. Scale bar represents $20 \mu \mathrm{m}$. 


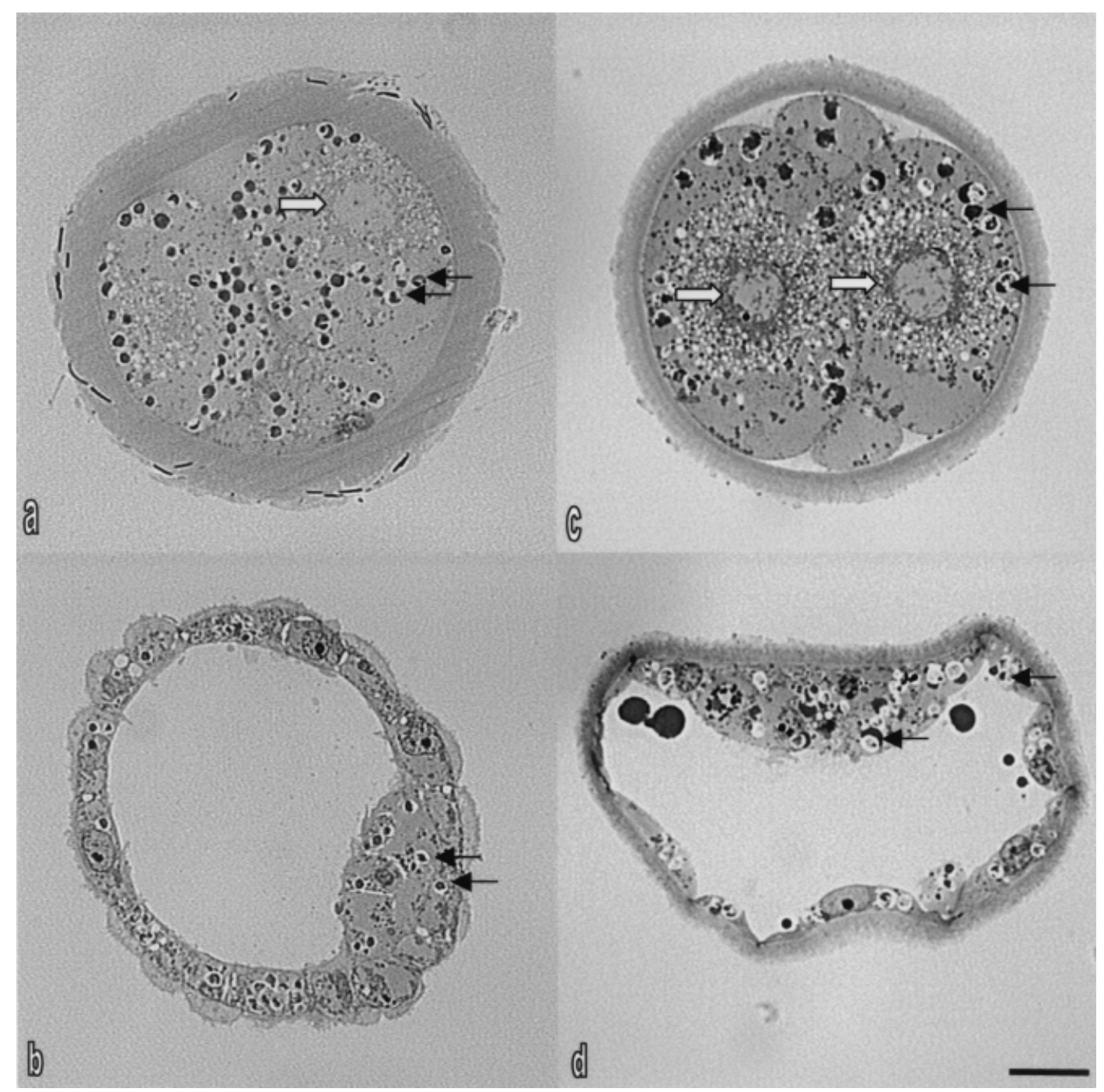

Figure 2 Light micrographs of semi-thin sections of porcine early embryos 2 days $(a, c)$ and $5-6$ days $(b, d)$ post-fertilisation, developed in vivo $(a, b)$ or in vitro $(c, d)$, showing the localisation of lipid droplets (small arrows) in the blastomeres. Note the more homogeneous, dense aspect of lipid deposited in the in vivo-developed embryos compared with the larger droplets in the in vitro embryos. White arrows, nucleus. Scale bar represents $20 \mu \mathrm{m}$.

\section{Discussion}

The mechanisms by which lipid droplets develop or degenerate are not well understood. During the development of lipid droplets in adipocytes, it is reported that a number of proteins, such as perilipins (perilipin A, B and C) or caveolin/oleosin, play an important role in the formation of the droplets as lipid deposits (Brown, 2001). However, the mechanism behind the degradation of lipid droplets has not been reported in any cell, including oocytes or embryos. Lipid droplets serve as storage depots for neutral lipids such as triglyceride and sterol esters (Brown, 2001). It may be possible that the morphological changes in lipid droplets observed during fertilisation and early embryonic development depend on the energy status in these cell types, because the density of the droplets after osmium fixation varies depending on the degree of saturation of triglycerides (Abe et al., 1999a). In the present study, the density of the lipid droplets in the oocytes matured both in vivo and in vitro decreased just after fertilisation and was restored again at the pronuclear stage. These results emphasise that energy consumption is accelerated for the completion of the early stage of fertilisation, where numerous nuclear and cytoplasmic changes occur such as membrane fusion with the sperm surface, cortical granule reaction, replacement of sperm nuclear protamines (Shimada et al., 2000) by histones (Nakazawa et al., 2002), sperm head decondensation, pronuclear membrane formation, replication of gamete DNA, etc. Energy substrates for the completion of these changes 


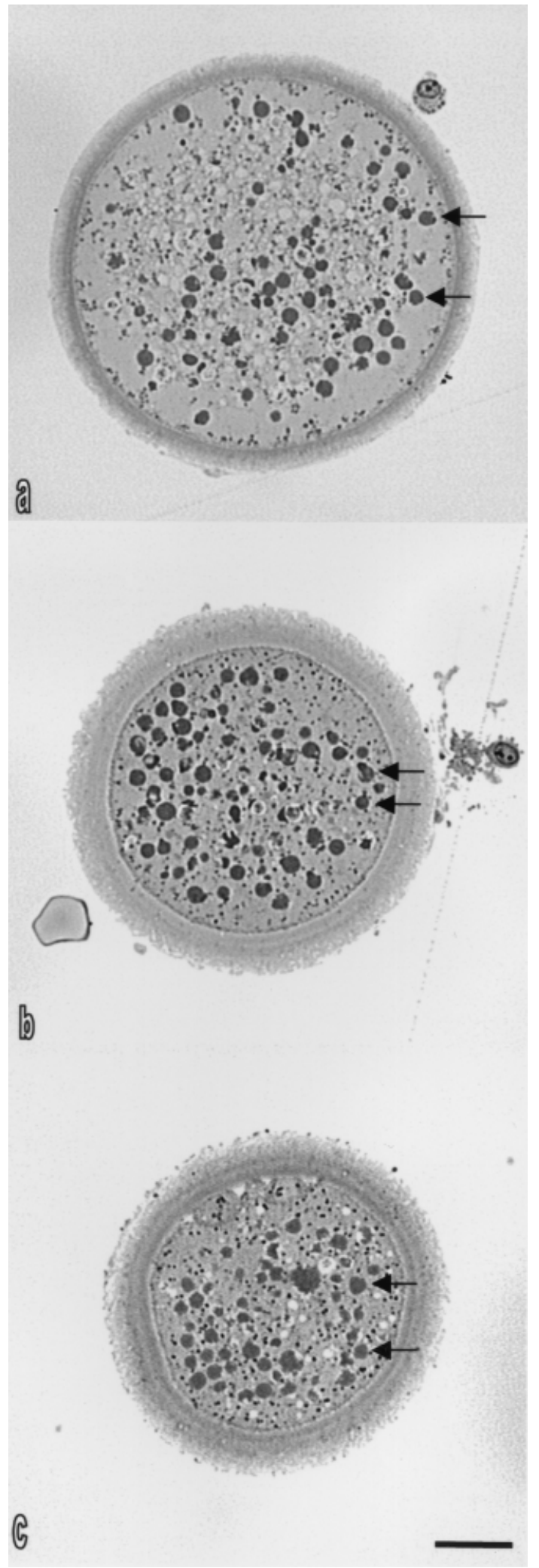

Figure 3 Light micrographs of semi-thin sections of porcine in vitro-matured oocytes submitted to electrostimulation and fixed for TEM at $3 \mathrm{~h}(a), 10 \mathrm{~h}(b)$ and $20 \mathrm{~h}(c)$ thereafter. Lipid droplets (small arrows) have the same appearance throughout. Scale bar represents $20 \mu \mathrm{m}$. may be related to changes in cytoplasmic lipid storage. It has been reported that, in toad eggs, fertilisation triggers a decrease in triglycerides and diglycerides while, in contrast, free fatty acid increases continuously (Alonso et al., 1986). This report suggests that the decrease in triglycerides is a reflection of the activation of lipolytic enzymes and the subsequent oxidation of fatty acids to meet the increasing metabolic energy requirements brought on by external fertilisation. Although the report was not from mammalian species, it is possible that mammalian oocytes also undergo such dramatic changes during internal fertilisation. After the storage of cytoplasmic lipids for fertilisation, morphological and functional changes may occur in the appearance and distribution of lipid droplets. At the pronuclear stage, enough energy is supplied from the droplets to accomplish the events of fertilisation; on the other hand, lipid droplet storage may be restored again by the development of new lipid droplets.

In contrast to the fertilised oocytes, it is interesting that the oocytes stimulated parthenogenetically did not show any such dramatic change. The reason is yet to be clarified. Oocyte activation seems to differ between fertilisation and parthenogenesis. From the point of view of the cell cycle, both fertilisation (Kikuchi et al., 1999b) and parthenogenesis (Kikuchi et al., 1995) induce a common cytoplasmic change, an inactivation of M-phase promoting factor, which results in nuclear progression from $\mathrm{M}$-phase to interphase. However, other cytoplasmic aspects do not seem to be completed in parthenogenesis. For example, the cortical reaction is affected by the activation method (Sun et al., 2001), and electric pulses can induce nuclear activation but not the cortical reaction in some species (Gulyas, 1980). In the present study, electric pulses were used to induce nuclear activation, resulting in more than $80 \%$ of stimulated oocytes forming a female pronucleus. However, most of the activated oocytes still contained a relatively large amount of cortical granules even at $10 \mathrm{~h}$ after the stimulation when they were evaluated by both semi-thin (Fig. 3b) and ultra-thin (Fig. 6b) sections. The method of parthenogenetic stimulation used in the present does not seem to induce full cytoplasmic activation, resulting in the consumption of only a small amount of the oocyte's energy source, and this may explain why the morphology of the lipid droplets did not change dramatically even after nuclear activation.

It is reported that the cytoplasm of bovine embryos produced in vivo or in vitro contains numerous lipid droplets prior to the blastocyst stage (Plante \& King, 1994; Abe et al., 2002) and, as bovine embryos develop, excess lipid may be sequestered within the cell and utilised by mitochondria for the increased production of ATP (Stojkovic et al., 2001) required for blastocoele 


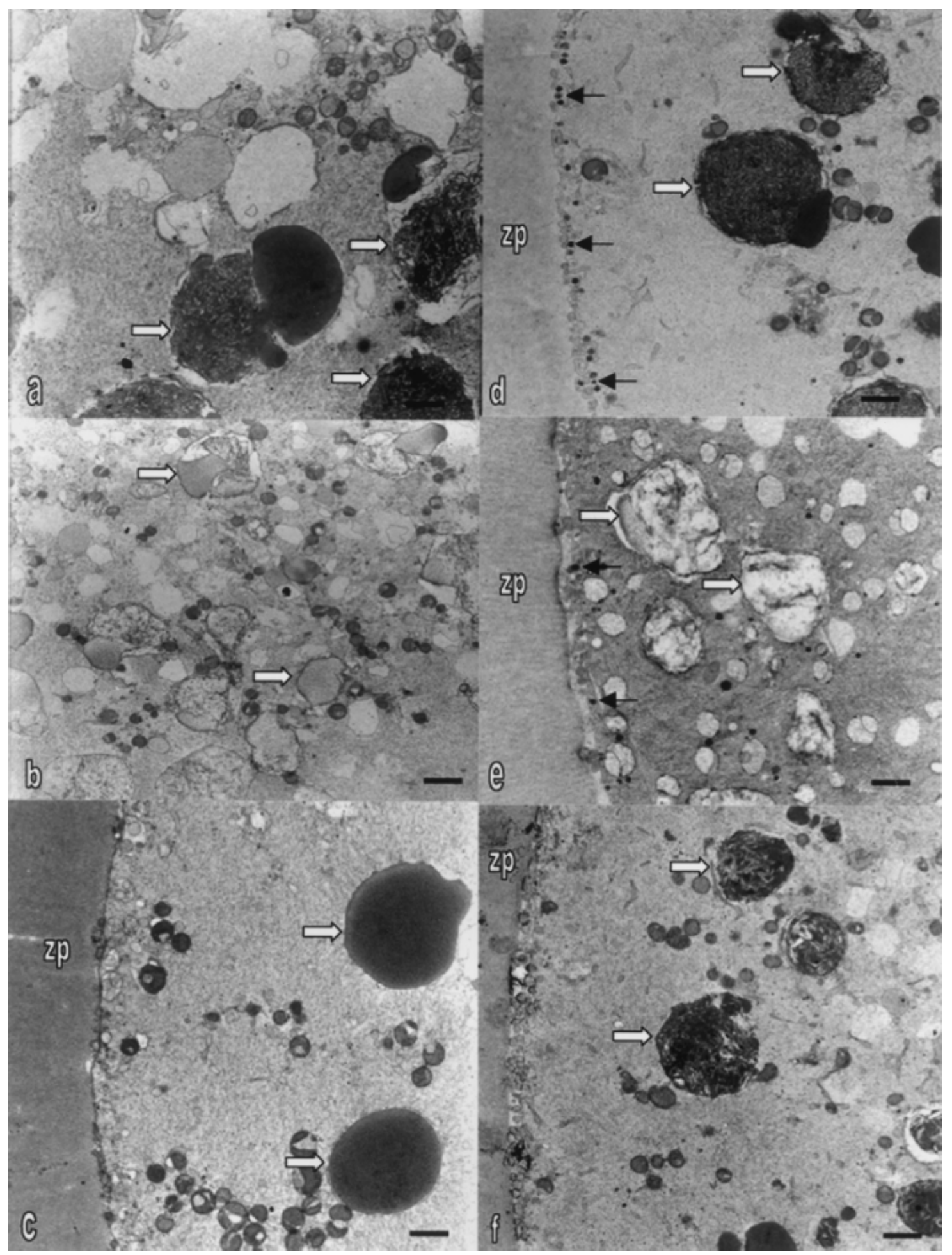

Figure 4 Transmission electron (TEM) micrographs of the cortical cytoplasm of porcine oocytes in vivo ( $a-c)$ or in vitro $(d-f)$ matured $(a, d)$ fertilised $(b, e)$ or in pronuclear stage $(c, f)$ depicting the appearance of the lipid droplets (white arrows). Note the more homogeneous, electron-dense appearance of the lipid droplets in the in vivo-developed oocytes. zp, zona pellucida; small arrows, cortical granules. Scale bar represents $5 \mu \mathrm{m}$.

formation or differentiation of cell lineages (Sathananthan \& Trounson, 2000; Crosier et al., 2001). However, a certain amount of lipid droplets still exist at the blastocyst stage in bovine (Abe et al., 1999a, $b$,
2002; Crosier et al., 2001) and also porcine (in the present study) embryos. Although lipid droplets were present in the blastocyst stage in both species, their morphological characteristics seem to be different. In 


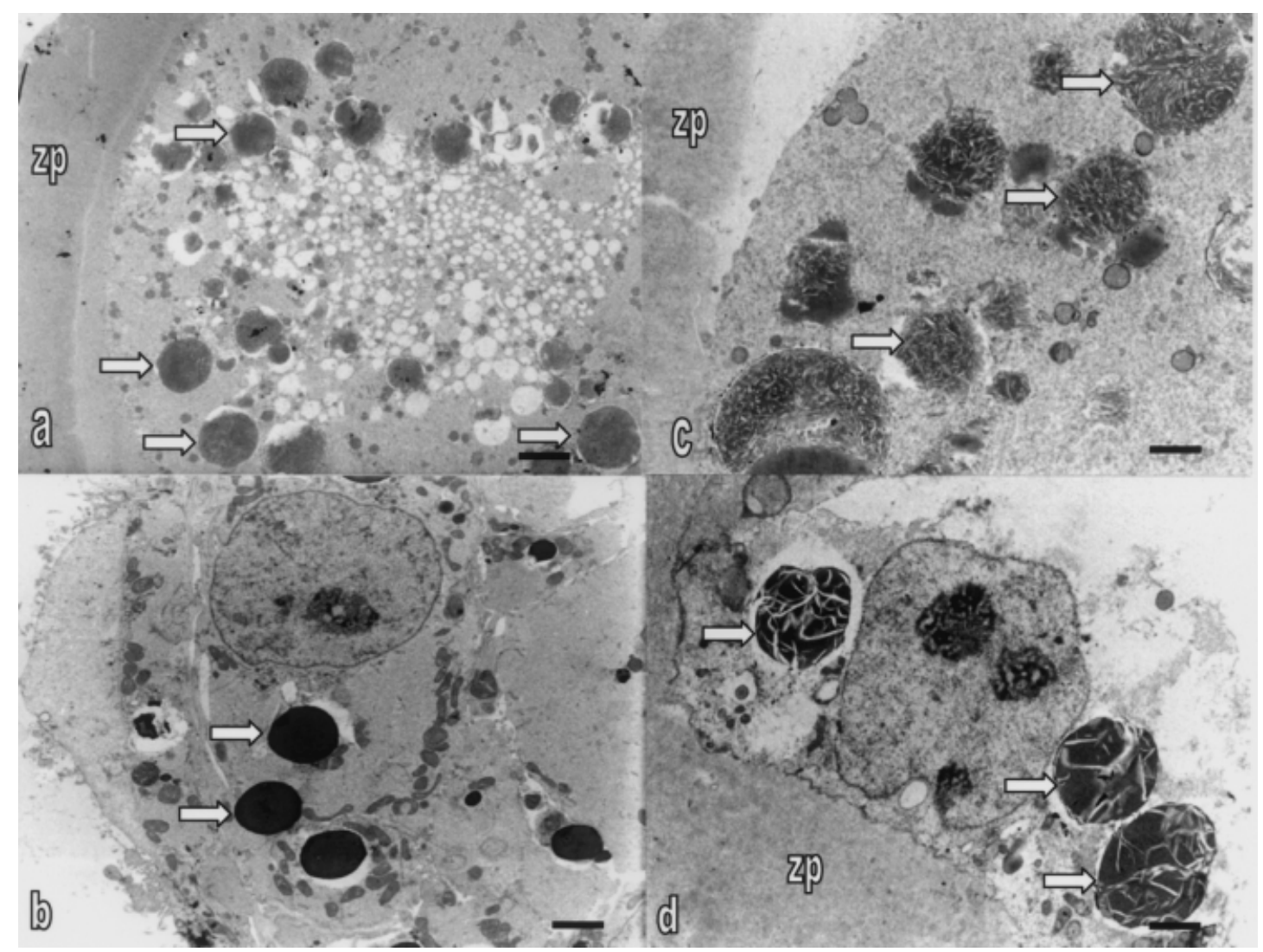

Figure 5 Transmission electron micrographs of porcine early embryos 2 days $(a, c)$ and 5-6 days $(b, d)$ post-fertilisation, developed in vivo $(a, b)$ or in vitro $(c, d)$, depicting the appearance of the lipid droplets (white arrows) in the blastomeres. Note the more homogeneous, electron-dense appearance of the lipid deposits in the in vivo-developed embryos, as seen previously in the oocytes. zp, zona pellucida. Scale bar represents $5 \mu \mathrm{m}(a, b)$ or $10 \mu \mathrm{m}(c, d)$.

bovine morulae and blastocysts, lipid droplets are homogeneous or fully saturated, showing a similar electron density in in vivo- or in vitro-produced embryos (Crosier et al., 2001). Sometimes, lipid droplets have been partially saturated in appearance in both in vivo and in vitro embryos, and associated with lysosome-like vesicles (Mohr \& Trounson, 1981; Abe et $a l ., 1999 a, b)$. In addition, the number of lipid droplets in the in vitro blastocysts, cultured in a serum-containing medium, is higher than that in the in vivo-developed embryos (Abe et al., 1999a, b). In contrast, in porcine embryos, the electron density of the lipid droplets present in in vivo embryos was homogeneous or fully saturated, while lipid droplets in in vitro-produced embryos appeared partially saturated (Fig. $4 a-d)$. Lysosome-like vesicles were not apparent in either type of porcine specimen. These changes in the appearance of the lipid droplets in porcine embryos began to be observed at the pronuclear stage, and became fully apparent by the 2- to 4-cell and blastocyst stages, suggesting that the density of lipid droplets seems to be fully restored within the homogeneous appearance in the in vivo specimens but becomes only partially saturated in the in vitro specimens. The func- tional aspect of lipid saturation, which also was observed in a proportion of the droplets seen in matured oocytes, is not well understood, although the differences seen by TEM between in vivo and in vitro specimens are quite interesting. In bovine morulae or blastocysts, the homogeneity or saturated appearance of lipid droplets increases when they are cultured in serum-supplemented medium (Abe et al., 1999a, b, 2002) suggesting the incorporation of triglyceride (Ferguson \& Leese, 1999) or lipoprotein (Sata et al., 1999) from serum. Our IVC medium for porcine zygotes is supplemented with BSA (Fraction V), which can be combined with fatty acid. This may cause an incomplete uptake of apoplipoprotein (the carrier protein for neutral lipid, phospholipid or cholesterol) (Abe et al., 2000), due to the absence of serum in the porcine culture medium. In addition, lysosome-like vesicles are observed less frequently, especially in bovine blastocysts cultured in serum-supplemented medium, leaving a question mark as regards their function in relation to lipid deposits (Abe et al., 1999a, b). Lysosome-like vesicles were not observed in either in vivo or in vitro porcine embryos.

The IVC system used in the present study has 


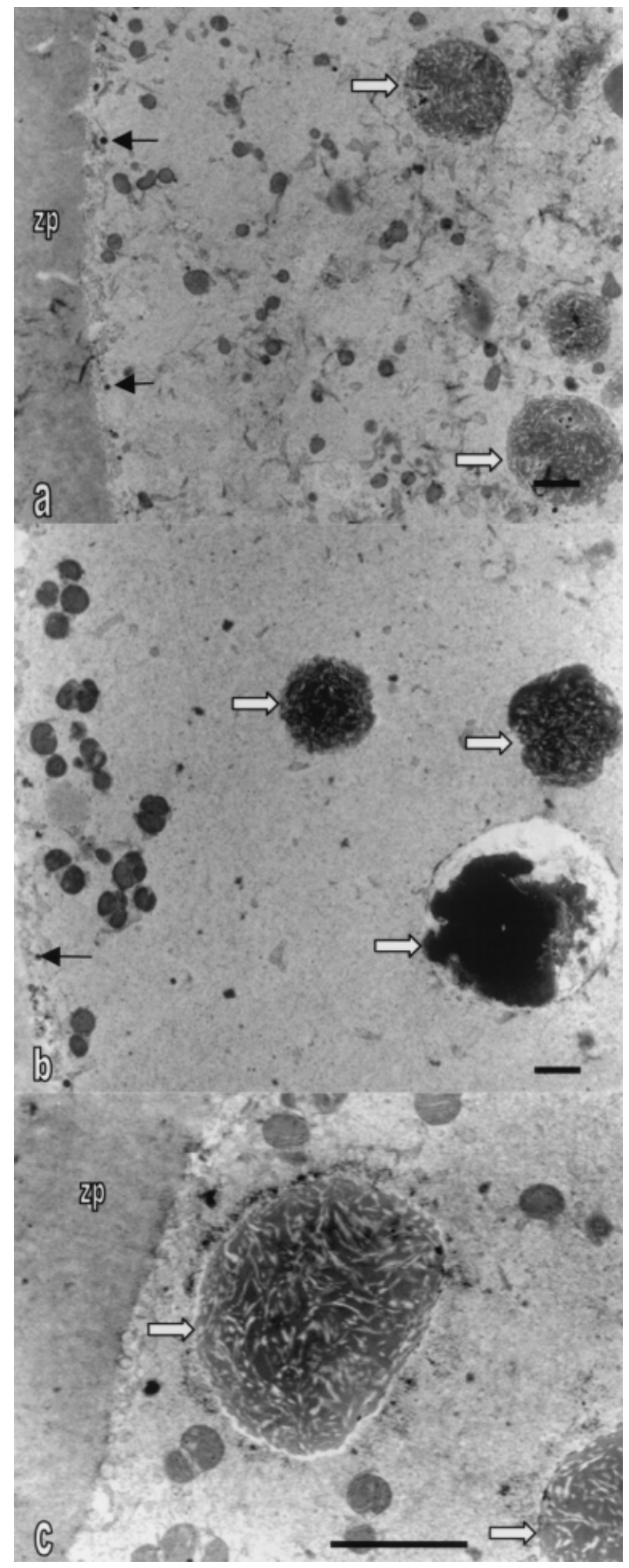

Figure 6 Transmission electron micrographs of the cortical cytoplasm of porcine in vitro-matured oocytes submitted to electrostimulation and fixed for TEM at $3 \mathrm{~h}(a), 10 \mathrm{~h}(b)$ and 20 $\mathrm{h}(c)$ thereafter. Note the appearance of the lipid droplets (white arrows) and the presence of cortical granules (small arrows) up to $10 \mathrm{~h}$ after electrostimulation. $\mathrm{zp}$, zona pellucida. Scale bar represents $5 \mu \mathrm{m}$. proved to have an advanced ability for in vitro blastocyst formation (average number of total cells of expanded blastocysts on day $6=86$ ). Following transfer to recipients, the blastocysts developed to piglets (Kikuchi et al., 2002). The present results suggest that there is a different morphology (perhaps reflected in a different function) of the lipid deposits in in vitro porcine embryos compared with in vivo embryos. In bovine embryos, an excess accumulation of lipid droplets is considered to be abnormal when they are cultured in serum-supplemented media (Abe et al., 1999a), which relates to the viability of frozen and thawed embryos (Yamashita et al., 1999; Abe et al., 2002). However, considering that the in vivo porcine embryos have a more homogeneous content of lipid droplets, the accumulation of these lipid droplets seems to be normal for further embryonic development in pigs.

In conclusion, the present study indicates marked variations in the morphology and amount of cytoplasmic lipid droplets during porcine oocyte maturation and fertilisation, as well as in preimplantation embryos both in vivo and in vitro, perhaps in relation to differences in energy status during preimplantation development in pigs. Understanding these steps of lipid transition may provide clues for the optimisation of culture conditions or cryopreservation of porcine oocytes and/or embryos in this species.

\section{Acknowledgements}

This study was supported by FORMAS (the former Swedish Council for Research in Agriculture and Forestry [SFJR]) and the Swedish Foundation for International Co-operation in Research and Higher Education (STINT), Stockholm, through the STINT Fellowship Programme in Reproductive Biotechnology: Bilateral University Co-operation Programme between the SLU and Japan. The authors thank Dr Hiroyuki Abe for critical discussions; Drs Takashi Nagai, Hiroaki Funahashi, Keita Suzuki and Dai-ichiro Fuchimoto for performing experiments; and Mrs Åsa Jansson, Teruko Aoki, Eri Yamauchi, Mamiko Sakurai and Mie Irie for technical assistance.

\section{References}

Abe, H., Yamashita, S., Itoh, T., Satoh, T. \& Hoshi, H. (1999a). Ultrastructure of bovine embryos developed from in vitromatured and -fertilized oocytes: comparative morphological evaluation of embryos cultured either in serum-free medium or in serum-supplemented medium. Mol. Reprod. Dev. 53, 325-35.

Abe, H., Otoi, T., Tachikawa, S., Yamashita, S., Satoh, T. \& Hsohi, H. (1999b). Fine structure of bovine morulae and blastocysts in vivo and in vitro. Anat. Embryol. 199, 519-27. 
Abe, H., Yamashita, S., Sata, R., Tsujii, H., Sato, T. \& Hoshi, H. (2000). Culture systems for efficient production of high quality bovine embryos developed from in vitromatured and -fertilized oocytes: quality evaluations of bovine embryos cultured in different culture systems using serum-free or serum-containing media (in Japanese with English abstract). Tissue Cult. Res. Commun. 19, 17-27.

Abe, H., Yamashita, S., Satoh, T. \& Hoshi, H. (2002). Accumulation of cytoplasmic lipid droplets in bovine embryos and cryotolerance of embryos developed in different culture systems using serum-free or serum-containing media. Mol. Reprod. Dev. 61, 57-66.

Alonso, T.S., Bonini de Romanelli, I.C. \& Bazan, N.G. (1986). Changes in triacylglycerol, diacylglycerol and free fatty acids after fertilization in developing toad embryos. Biochim. Biophys. Acta 875, 465-72.

Brown, D.A. (2001). Lipid droplets: proteins floating on a pool of fat. Curr. Biol. 11, R446-9.

Crosier, A.E., Farin, P.W., Dykstra, M.J., Alexander, J.E. \& Farin, C.E. (2001). Ultrastructural morphometry of bovine blastocysts produced in vivo or in vitro. Biol. Reprod. 64, 1375-85.

Dobrinsky, J.R. (2002). Advancements in cryopreservation of domestic animal embryos. Theriogenology 57, 285-302.

Ferguson, E.M. \& Leese, H.J. (1999). Triglyceride content of bovine oocytes and early embryos. J. Reprod. Fertil. 116, 373-8.

Funahashi, H., Cantley, T.C. \& Day, B.N. (1997). Synchronization of meiosis in porcine oocytes by exposure to dibutyryl cyclic adenosine monophosphate improves developmental competence following in vitro fertilization. Biol. Reprod. 60, 336-40.

Gulyas, B.J. (1980). Cortical granules of mammalian eggs. Int. Rev. Cytol. 63, 357-92.

Hyttel, P. \& Niemann, H. (1990). Ultrastructure of porcine embryos following development in vitro versus in vivo. Mol. Reprod. Dev. 27, 136-44.

Kikuchi, K., Nagai, T., Motlik, J., Shioya, Y. \& Izaike, Y. (1993). Effect of follicle cells on in vitro fertilization of pig follicular oocytes. Theriogenology 39, 593-9.

Kikuchi, K., Izaike, Y., Noguchi, J., Furukawa, T., Daen, F.P., Naito, K. \& Toyoda, Y. (1995). Decrease of histone H1 kinase activity in relation to parthenogenetic activation of pig follicular oocytes matured and aged in vitro. J. Reprod. Fertil. 105, 325-30.

Kikuchi, K., Nagai, T., Kashiwazaki, N., Ikeda, H., Noguchi, J., Shimada, A., Soloy, E. \& Kaneko, H. (1998). Cryopreservation and ensuing in vitro fertilization ability of boar spermatozoa from epididymides stored at $4{ }^{\circ} \mathrm{C}$. Theriogenology 50, 615-23.

Kikuchi, K., Kashiwazaki, N., Noguchi, J., Shimada, A., Takahashi, R., Hirabayashi, M., Shino, M., Ueda, M. \& Kaneko, H. (1999a). Developmental competence, after transfer to recipients, of porcine matured, fertilized, and cultured in vitro. Biol. Reprod. 60, 336-40.

Kikuchi, K., Naito, K., Noguchi, J., Simada, A., Kaneko, H., Yamashita, M., Tojo, H. \& Toyoda, Y. (1999b). Inactivation of p34 ${ }^{\text {cdc2 }}$ kinase by the accumulation of its phosphorylated forms in porcine oocyte matured and aged in vitro. Zygote 7, 173-9.
Kikuchi, K., Onishi, A., Kashiwazaki, N., Iwamoto, M., Noguchi, J., Kaneko, H., Akita, H. \& Nagai, T. (2002). Successful piglet production after transfer of blastocysts produced by a modified in vitro system. Biol. Reprod. 66, 1033-41.

Kim, J.Y., Kinoshita, M., Ohnishi, M. \& Fukui, Y. (2001). Lipid and fatty acid analysis of fresh and frozen-thawed immature and in vitro matured bovine oocytes. Reproduction 122, 131-8.

Leibo, S.P. \& Loskutoff, N.M. (1993). Cryobiology of in vitroderived bovine embryos. Theriogenology 39, 81-94.

Mburu, J.N., Einarsson, S., Dalin, A.-M. \& RodriguezMartinez, H. (1995). Ovulation as determined by transrectal ultrasonography in multiparous sows: relationships with oestrous symptoms and hormonal profiles. J. Vet. Med. 42, 285-92.

McEvoy, T.G., Coull, G.D., Broadbent, P.J., Hutchinson, J.S.M. \& Speake, B.K. (2000). Fatty acid composition of lipid in immature cattle, pig and sheep oocytes with intact zona pellucida. J. Reprod. Fertil. 118, 163-70.

Mohr, L.R. \& Trounson, A.O. (1981). Structural changes associated with freezing of bovine embryos. Biol. Reprod. 25, 1009-25.

Nagai, T., Takahashi, T., Masuda, H., Shioya, Y., Kuwayama, M., Fukushima, M., Iwasaki, S. \& Handa, A. (1988). In-vitro fertilization of pig oocytes by frozen boar spermatozoa. J. Reprod. Fertil. 84, 585-91.

Nagasima, H., Kashiwazaki, N., Ashman, R.J., Grupen, C.G. \& Nottle, M.B. (1995). Cryopreservation of porcine embryos. Nature 374, 416.

Nakazawa, Y., Shimada, A., Noguchi, J., Domeki, I., Kaneko, H. \& Kikuchi, K. (2002). Replacement of nuclear protein by histone in pig sperm nuclei during in vitro fertilization. Reproduction 124, 565-72.

Petters, R.M. \& Wells, K.D. (1993). Culture of pig embryos. J. Reprod. Fertil. Suppl. 48, 61-73.

Plante, L. \& King, W.A. (1994). Light and electron microscopic analysis of bovine embryos derived by in vitro and in vivo fertilization. J. Assist. Reprod. Genet. 11, 515-29.

Sata, R., Tsujii, H., Abe, H., Yamashita, S. \& Hoshi, H. (1999). Fatty acid composition of bovine embryos cultured in serum-free and serum-containing medium during early embryonic development. J. Reprod. Dev. 45, 97-103.

Sathanathan, A.H. \& Trounson, A.O. (2000). Mitochondrial morphology during preimplantational human embryogenesis. Hum. Reprod. 15 (Suppl. 2), 148-59.

Shimada, A., Kikuchi, K., Noguchi, J., Akama, K., Nakano, M. \& Kaneko, H. (2000). Protamine dissociation before decondensation of sperm nuclei during in vitro fertilization of pig oocytes. J. Reprod. Fertil. 120, 247-56.

Simonsson, A. (1994). Näringsrekommendationer och fodermedel till svin. Swedish University of Agricultural Sciences, Research Information Centre, Husdjur. 75, 71.

Stojkovic, M., Machado, S.A., Stojkovic, P., Zakhartchenko, V., Hutzler, P., Goncalves, P.B. \& Wolf, E. (2001). Mitochondrial distribution and adenosine triphosphate content of bovine oocytes before and after in vitro maturation: correlation with morphological criteria and developmental capacity after in vitro fertilization and culture. Biol. Reprod. 64, 904-9. 
Sun, Q.Y., Lai, L.L., Park, K.W., Kuhholzer, B., Prather, R.S. \& Schatten, H. (2001). Dynamic events are differently mediated by microfilaments, microtubules, and mitogen-activated protein kinase during porcine oocyte matured and fertilized in vitro. Biol. Reprod. 64, 879-89.

Suzuki, H., Eriksson, B., Shimizu, H., Nagai, T. \& RodriguezMartinez, H. (2000). Effect of hyaluronan on monospermic penetration of porcine oocytes fertilized in vitro. Int. J. Androl. 23, 13-21.
Suzuki, K., Asano, A., Eriksson, B., Niwa, K., Nagai, T. \& Rodriguez-Martìnez, H. (2002). Capacitation status and in vitro fertility of boar spermatozoa: effects of seminal plasma, cumulus-oocyte-complexes-conditioned medium and hyaluronan. Int. Androl. 25, 84-93.

Yamashita, S., Abe, H., Itoh, T., Satoh, T. \& Hoshi, H. (1999). A serum-free culture system for efficient in vitro production of bovine blastocysts with improved viability after freezing and thawing. Cytotechnology 31, 121-9. 